Décadrages Décadrages

cınéma, a travers champs Cinéma, à travers champs

19 | 2011

Autour d'Elephant de Gus Van Sant

\title{
Elephant : misère de l'adolescence dans une modernité en crise
}

Alice Laguarda

\section{(2) OpenEdition}

Journals

Édition électronique

URL : http://journals.openedition.org/decadrages/304

DOI : $10.4000 /$ decadrages.304

ISSN : 2297-5977

Éditeur

Association Décadrages

Édition imprimée

Date de publication : 10 octobre 2011

Pagination : 21-29

ISBN : 978-2-9700668-3-5

ISSN : 2235-7823

Référence électronique

Alice Laguarda, «Elephant : misère de l'adolescence dans une modernité en crise », Décadrages [En

ligne], 19 | 2011, mis en ligne le 10 octobre 2012, consulté le 19 avril 2019. URL : http://

journals.openedition.org/decadrages/304; DOI : 10.4000/decadrages.304

(B) Décadrages 


\section{Elephant: misère de l'adolescence \\ dans une modernité en crise}

\section{par Alice Laguarda}

Le cinéma européen a vu naître dans les années 1960 une catégorie d'œuvres nourries du double héritage culturel moderne et romantique: «incommunicabilité» et variations sur le motif du désert chez Michelangelo Antonioni, road movie et méditations mélancoliques sur l'imaginaire du cinéma américain chez Wim Wenders, interrogations sur la fonction de l'art et sur l'identité des personnages chez Jean-Luc Godard. De telles œuvres sont les manifestations des bouleversements et des contradictions que ce double héritage porte en lui et de leurs répercussions morales et esthétiques. A partir d'un scénario inspiré d'un fait divers (la fusillade du lycée de Columbine en 1999, dans les environs de la ville de Littleton) qui relate la planification et l'assassinat de lycéens par deux adolescents, Gus Van Sant a construit un film qui s'inscrit dans la continuité de ce cinéma européen, fondé sur l'imminence du chaos, au cœur duquel règnent indéfinition et suspension. Le cinéaste questionne dans Elephant (USA, 2003) la représentation de la violence,

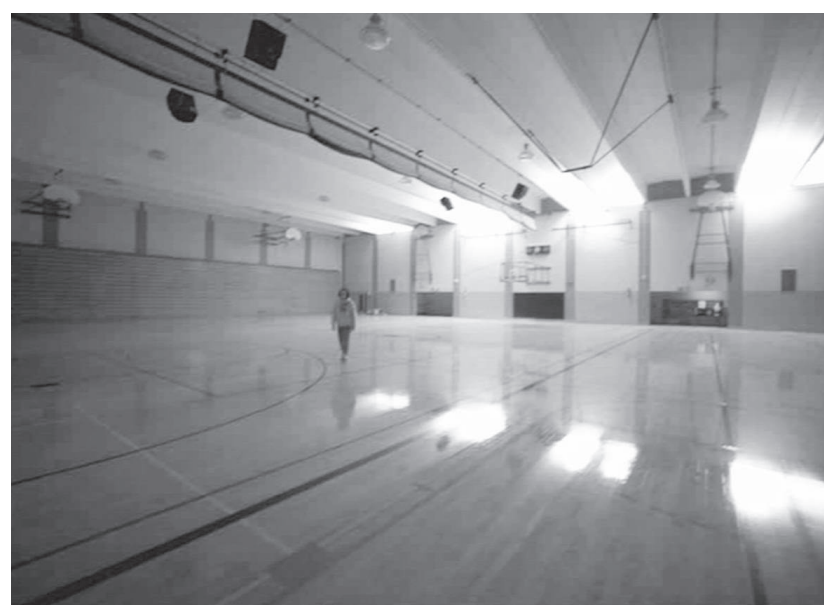


1 Voir Hannah Arendt, Condition de l'homme moderne, Paris, Calmann-Lévy, trad. française Georges Fradier, 1961 [1958]; Robert Legros, L'idée d'humanité, Paris, Grasset, 1990.

2 Martin Heidegger, Lettre sur l'humanisme, Paris, Aubier, trad. française Roger Munier, 1964 , p. 81. réitérant la tension entre raison et irrationnel qui caractérise la longue crise des sociétés modernes.

\section{Le monde indéfini}

Dans Elephant, tout semble comme suspendu. La suspension est d'abord liée à la spécificité des personnages: des adolescents. On ne connaît pas leur histoire personnelle ni leur cheminement psychologique. Ils apparaissent comme isolés en eux-mêmes, errant dans des espaces trop grands. Cette dimension fantomatique se traduit par l'utilisation de longs travellings avant dans lesquels on suit les personnages de dos ou de travellings arrière les présentant de face, déambulant dans les différents espaces du lycée. Ils ne semblent partager aucune réalité, aucun monde commun. Cet isolement met en avant le paradoxe des sociétés modernes, fondées sur le désir de relation avec l'autre, sur le désir de communication, mais au sein desquelles ne subsistent que des logiques de séparation. Des sociétés où les individus sont comme des éléments séparés les uns des autres, pris dans le cercle de l'incommunicabilité. Une telle représentation rejoint l'interrogation philosophique sur l'humanité de l'homme qui, de Hannah Arendt à Robert Legros ${ }^{1}$, a accompagné la crise des sociétés modernes occidentales tout au long du $\mathrm{XX}^{\mathrm{e}}$ siècle. Selon Martin Heidegger, la crise de la modernité s'explique notamment par la domination d'une conception de l'homme à partir de "l'animalitas» et non en fonction de son humanitas. Cela a pour conséquence que l'homme "méconnaît d'abord le plus proche et se tient à ce qui vient après", et oublie "la vérité de l'Etre au profit d'une invasion de l'étant non pensé dans son essence» 2 . L'Etre heideggerien symbolise la promesse d'un accès à la compréhension du monde, au sens. Mais l'étant semble s'y être substitué, fantôme d'Etre, expression d'une impuissance tragique à sentir, comprendre, exister. Les adolescents d'Elephant incarnent parfaitement ce sentiment de perte, cet oubli de soi.

La figure de l'adolescent est récurrente dans le parcours cinématographique de Gus Van Sant. Dans My ozwn Private Idaho (USA, 1991) par exemple, un couple de jeunes hommes erre entre différentes villes des Etats-Unis et d'Italie; Paranoid Park (USA, 2006) est centré sur un personnage d'adolescent mêlé à un crime accidentel. Dans ces œuvres, les personnages sont présentés comme des individus marginalisés par leurs désirs, leurs actes et la société des adultes. Or, dans la littérature romantique, l'adolescent symbolise l'indéfinition, l'ambivalence sexuelle et morale, la proie idéale du conflit entre passions et raison. Mais la figure de l'adolescent est aussi une invention de la modernité du $\mathrm{XX}^{\mathrm{e}}$ siècle. 
L'adolescent crée sa propre culture, avec par exemple le rock n'roll ou la techno, et les modes vestimentaires et rites collectifs qui les accompagnent. Devenu un consommateur, il revendique ses propres aspirations, sa propre identité sociale et culturelle, et refuse d'être intégré dans la société des adultes. Les adolescents d'Elephant semblent écartelés entre ces deux représentations culturelles, entre désir d'affirmation de soi, d'autonomie, et enfermement dans le moi intérieur, à la frontière de l'autisme. Cela renforce le sentiment d'un malaise dans l'identification de l'adolescence comme un monde fluctuant et fragile. Le monde des deux tueurs Alex et Eric est ainsi traversé d'un ensemble de clichés, références non comprises et banalisées qu'ils consomment parmi d'autres. Ils arrivent au lycée habillés de tenues militaires pour exécuter leur plan, visionnent chez eux des films sur la période nazie. Gilles Deleuze, dans ses ouvrages consacrés au cinéma, a mis en avant l'apparition dans les années 1960-70 d'une nouvelle typologie de personnages aux identités incertaines, aux psychologies souvent déstructurées, incapables de distinguer la frontière entre Bien et Mal. Le philosophe identifie un moment historique et esthétique particulier à partir de la thématique de la "crise de l'image-action". Les personnages qui correspondent à cette catégorie partagent avec ceux d'Elephant certaines caractéristiques de représentation:

"Ce sont ces images flottantes, ces clichés anonymes qui circulent dans le monde extérieur mais aussi pénètrent chacun et constituent son monde intérieur, si bien que chacun ne possède en soi que des clichés psychologiques par lesquels il pense et il sent, se pense et se sent, étant lui-même un cliché parmi les autres dans le monde qui l'entoure [...] Pour que les gens se supportent eux-mêmes et le monde, il faut que la misère ait gagné l'intérieur des consciences.»3

Le cinéma se fait alors le miroir d'une société moderne dominée par la misère affective, psychologique et sociale. L'atmosphère d'Elephant peut être rapprochée de la réflexion de Gilles Deleuze: les zones floues dans certains plans, les personnages à "interférences faibles", tour à tour principaux et secondaires, composent un monde inhumain, abstrait.

\section{L'union des contraires}

Elephant est marqué par le sentiment d'un chaos imminent. Le film s'ouvre sur un plan de ciel menaçant qui s'obscurcit totalement et se clôt par un même type de plan d'un ciel envahi de nuages denses et tourbillonnants, nous suggérant que le drame auquel nous assistons est relié aux forces de la nature. Des adolescents apparemment tranquilles se transforment en tueurs sans pitié. Une nature domestiquée et
3 Gilles Deleuze, L'image-mouvement, Paris, Minuit (coll. "Critique"), 1983, p. 281. 
4 "So foul and fair a day I have not seen", dans Macbeth, acte I, scène 3 .

5 L'on peut s'accorder à identifier quelques traits récurrents du sublime dans l'art: immédiateté, instantanéité, irrationalité. Edmond Burke associe le sublime au terrible dans sa Recherche philosophique sur l'origine de nos idées du sublime et du beau (1757). Selon Baldine Saint Girons, il importe alors d'en comprendre le sens dans le contexte d'une "philosophie des passions": "Le sublime ébranle notre narcissisme et menace plus ou moins notre intégrité non seulement physique mais psychique et morale." Voir Baldine Saint Girons, "Le paysage et la question du sublime", dans $L e$ paysage et la question du sublime, cat. expo., Musée de Valence/RMN, 1997, p. 80.

6 A ce propos, voir l'article de Gaspard Vignon dans le présent dossier. apaisante (l'environnement immédiat du lycée) devient le théâtre de l'inconcevable. Le conflit entre le beau et l'affreux, entre le rationnel et l'irrationnel, définit le climat esthétique du film. Le poids de la nature, la tension entre ordre et chaos, typiques de la vision romantique du monde, dominent. L'un des deux adolescents tueurs prononce ainsi la phrase: "Jamais je n'ai vu de jour si beau ni si affreux»4. Attribuée à Shakespeare, cette phrase peut également faire référence aux théories sur le sublime nées au XVIII ${ }^{\mathrm{e}}$ siècle et s'appliquant à la peinture de paysage (scènes de naufrages, d'incendies, de tempêtes en mer, esthétiques de la ruine) 5 .

Peu de temps avant le massacre, Alex et Eric se retrouvent dans la chambre de l'un d'eux. Tandis que l'un joue au piano la Lettre à Elise puis la Sonate au clair de Lune - l'une des œuvres dites "romantiques" de Beethoven 6 -, l'autre se distrait sur son ordinateur avec un jeu vidéo dans lequel il manipule une arme, tirant sur des hommes de dos, perdus dans un espace vide et gris. La séquence commence par un mouvement panoramique circulaire pour venir s'arrêter sur l'adolescent et son jeu vidéo. L'irrationnel et le cruel surgissent, indéchiffrables, bruts. Ils sont traités froidement, sans effets dramatiques ni compassion. Elephant nous parle d'une société qui vit l'effondrement des valeurs philosophiques modernes (liberté, dialogue, tolérance, relation, etc.) au profit d'un monde en proie à l'entropie et à l'absence de sens, rythmé par des débordements, des irruptions de violence. La dimension sublime du film est fondée sur l'association simultanée, chez le spectateur, d'un sentiment de terreur et d'une beauté qui trouble la perception et la raison et engendre une fascination ou une peur intense:

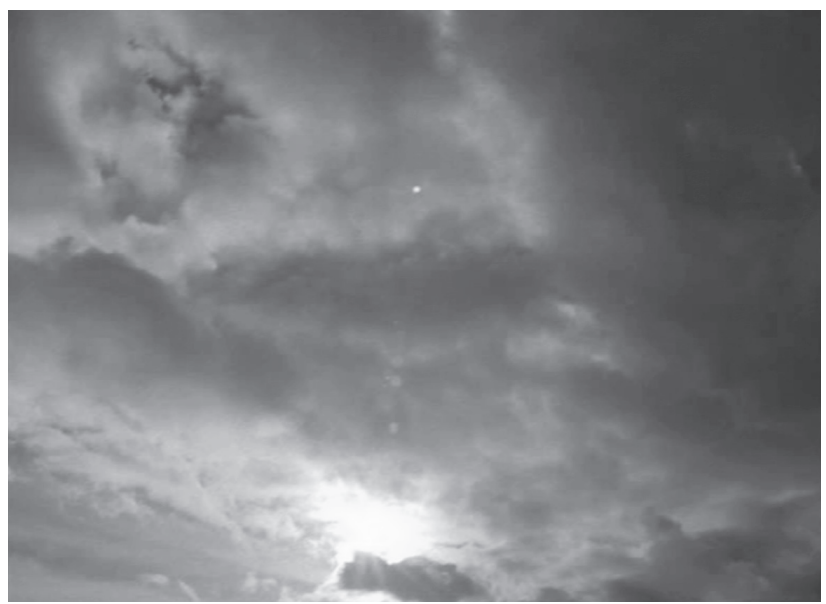


"L'art et la poésie antiques tendent à séparer rigoureusement ce qui est hétérogène, l'art et la poésie romantique se plaisent en revanche à des mélanges insolubles; toutes les oppositions [...] le souvenir et le pressentiment [...] le terrestre et le divin, la vie et la mort, fusionnent de la manière la plus intime. L'art et la poésie romantiques expriment l'aspiration mystérieuse au chaos qui lutte perpétuellement pour engendrer de nouvelles et merveilleuses créatures, chaos qui se dissimule sous la création ordonnée. » $\mathbf{7}$

Les références au sublime et au romantisme sont également présentes dans d'autres œuvres de Gus Van Sant comme Gerry (USA, 2002) et Last Days (USA, 2005). Des personnages de jeunes adultes aux identités tourmentées et indéfinies - les deux personnages masculins de Gerry, dont on ne sait s'il s'agit d'un seul et même être; le héros de Last Days, musicien de rock - sont pris au cœur d'un monde dont le sens leur échappe et qui, par la nature, va se révéler tour à tour destructeur et régénérateur. Dans ces films, la fonction des décors naturels est de signifier la coexistence de sentiments de perte ou de mort avec la possibilité d'une échappée vers un autre ordre symbolique et spirituel. Le désert dans Gerry est hostile, source d'hallucinations et de dangers physiques. Il peut être considéré comme une métaphore du "désert» de la société moderne: un monde de la déliaison entre les êtres, entre l'individu et son environnement. Mais il est aussi un espace initiatique dans lequel les personnages prennent conscience de leur destin, de leur présence au monde. La forêt dans Last Days est le repaire d'une nature maternelle et grandiose. C'est à la fois un lieu d'errance et un lieu clos, limité par des murailles d'arbres, promesse d'une possible élévation spirituelle et artistique pour le personnage principal: "Le sous-bois m'exclut de ce qui est au-delà de sa surface enveloppante, mais pas de n'importe quelle manière: d'un côté, il limite ma vision; de l'autre, il la renouvelle. » $\mathbf{8}$ L'analogie avec le «désert» conditionne aussi l'espace-temps d'Elephant. Le lycée est le lieu de l'oscillation entre humain et inhumain, physicalité et abstraction, ouverture et fermeture. C'est un «espace vidé» dont on dirait qu'il a absorbé «les personnages et les actions pour n'en garder qu'une description géophysique, un inventaire abstrait» 9 .

\section{Inquiétante musique}

La Sonate au clair de lune de Beethoven que joue l'un des lycéens tueurs et qui ouvre le film ainsi que la Lettre à Elise contribuent au climat contemplatif d'Elephant. La musique est un instrument de transcendance de l'existence matérielle, mais elle peut aussi être l'expression d'une libération chaotique des passions. Les recours à la musique romantique sont
7 Friedrich Schlegel, Fragments (1797), dans Charles Le Blanc, Laurent Margantin, Olivier Schefer (éd.), La forme poétique du monde. Anthologie du romantisme allemand, Paris, José Corti, 2003, p. 527.

8 Baldine Saint Girons, "Le paysage et la question du sublime", op. cit., p. 101.

9 Gilles Deleuze, L'image-temps, Paris, Minuit (coll. "Critique"), 1985, p. 12. 
10 Stéphane Delorme et Ariane Allemandi, livret Lycéens et apprentis au cinéma, Dossier Elephant, Paris, CNC Publications, septembre 2007; voir les travaux des artistes Laetitia Delafontaine et Grégory Niel dans le cadre du Centre de recherche et de création "La forme des idées", 2011: www.laformedes idees.net. innombrables dans l'histoire du cinéma. On trouve cette récurrence chez un autre cinéaste qui a placé la violence au cœur de la plupart de ses œuvres: Stanley Kubrick. Le parallèle a ainsi été effectué à plusieurs reprises entre Orange mécanique (A Clockwork Orange, GB, 1971) et Elephant 10. C'est que Kubrick et Van Sant interrogent tous deux le poids de la violence dans les sociétés modernes et la figure de l'adolescent comme proie privilégiée de ce phénomène. Chez Stanley Kubrick, tout est fantasme, exacerbation, outrance, déformation - que l'on pense au traitement des décors, au rythme du montage, aux angles de prise de vues, etc. La caméra s'accorde à la folie des personnages. Les personnages ne sont pas juste des êtres fantomatiques et désincarnés comme chez Gus Van Sant. Cependant, ni Kubrick ni Van Sant ne s'inscrivent dans une veine réaliste ou objective : chacun a créé un espace-temps plus fantasmé que réel. La dimension fantasmatique se nourrit chez Stanley Kubrick du personnage d'Alex et de sa perception déviante du monde qui l'entoure, un monde outré et chaotique. Dans A Clockwork Orange, la musique de Beethoven est ainsi détournée, parodiée, retravaillée avec un synthétiseur par le compositeur Walter Carlos. Elle contribue à la représentation d'un monde de l'irrévérence et de la violence gratuite, où rien ne semble pouvoir échapper à la destruction.

Dans le film de Gus Van Sant, le rapport au temps est plus lent, il repose sur la suspension et les boucles: l'utilisation de longs plansséquences et certaines séquences qui reprennent les mêmes situations vues selon les différents points de vue des personnages produisent un effet de boucle temporelle qui augmente les sentiments d'indéfinition, de perte de substance et de sens. Il n'y a pas de narration traditionnelle

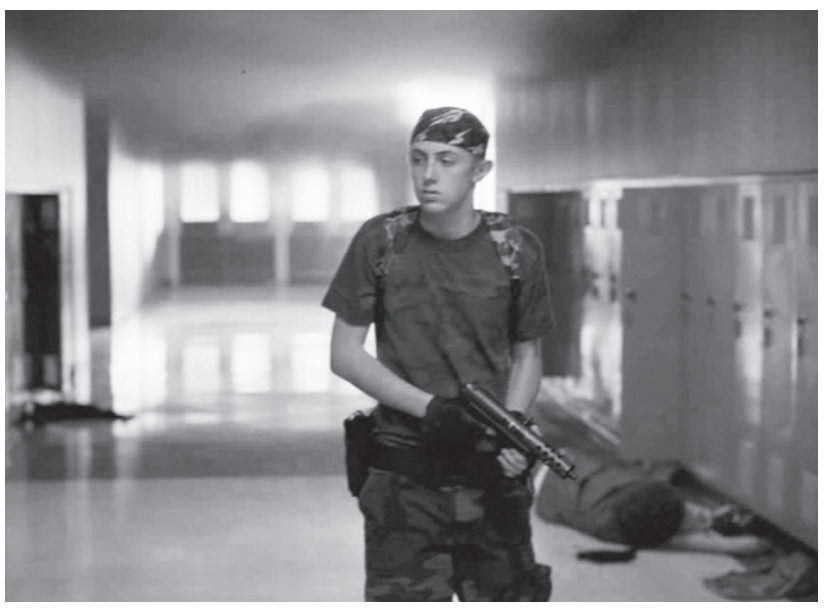


fondée sur des enchaînements logiques. Ce traitement du déroulement des faits engendre une temporalité élastique, malléable, non linéaire. La présence de la musique romantique dans certaines séquences contribue à instaurer un sentiment de torpeur, de fausse plénitude, qui rend l'irruption de la violence encore plus tragique, plus brutale, comme un point culminant de terreur.

\section{Failles de la modernité}

Dans Elephant, l'irrationnel surgit dans un monde rationnel dominé par la logique et les valeurs de la société moderne et explose au moment où les individus sont en phase d'éducation. C'est que la crise de la modernité est aussi et surtout une crise de l'éducation, et de l'autorité : deux grandes problématiques indispensables pour comprendre sur quoi se fonde la "crise». La relation et la communication sont fondatrices de la pensée moderne: c'est par elles en effet que se définit l'être humain, par le fait qu'il entre en relation avec l'autre. Or tout nous dit dans le film de Gus Van Sant qu'il y a une absence de communication entre les lycéens, mais aussi entre les générations, qui conduit à ce que tout soit considéré comme sans intérêt, chacun étant muré dans son intériorité. Le monde d'Elephant est trop lisse, les sentiments y sont refoulés. La fracture est profonde entre les parents et leurs enfants, entre les enfants et leurs éducateurs. Il n'y a pas d'intersubjectivité : les adolescents du lycée sont divisés en groupes ou "tribus» aux préoccupations éloignées les unes des autres (filles boulimiques anorexiques, fan de photo, adolescente complexée et mise à l'écart, couple d'amoureux, etc.). C'est que, contrairement à la grande promesse d'égalité du système américain, les adolescents se retrouvent "soumis à une autorité effrayante et vraiment tyrannique: la tyrannie de la majorité.»Il en résulte que les adolescents "ont été pour ainsi dire bannis du monde des adultes. Ils sont soit livrés à eux-mêmes, soit livrés à la tyrannie de leur groupe, contre lequel, du fait de sa supériorité numérique, ils ne peuvent se révolter»11. Les subjectivités semblent brisées, insaisissables. Cela est précisément traduit par l'écriture filmique: morcellement de séquences découpées par personnages (les passages correspondants sont introduits par des inserts indiquant le nom du personnage concerné), moments de suspension de l'action, dialogues presque insignifiants et livrés par bribes, personnages regardant vers le ciel... Tout semble noyé dans un même lointain: ici, "l'image ne renvoie plus à une situation globalisante ou synthétique, mais dispersive [...] la réalité est lacunaire» $\mathbf{1 2}$. C’est cette dispersion qui fait, aussi, que l'on ne s'attache pas aux personnages, qu'il n'y a pas de processus d'identification, mais une distance toujours maintenue entre
11 Hannah Arendt, La crise de la culture, chapitre "La crise de l'éducation ", Paris, Gallimard, trad. française Patrick Lévy, 1972, p. 233.

12 Gilles Deleuze, op. cit., p. 282. 
eux et nous. Cette dispersion va jusqu'à l'anéantissement: destruction symbolique et annonce du chaos lorsque les adolescents apparaissent à plusieurs reprises dans l'espace du lycée comme des points minuscules, perdus dans des espaces fonctionnels démesurément grands (salles de gym, de classe, réfectoire); anéantissement physique, lorsque le massacre final commence. C'est alors la tension entre fini et infini qui se manifeste, convoquant à nouveau le sentiment du sublime à travers la représentation d'une figure humaine isolée dans des espaces hors d'échelle, tiraillée entre pulsion de vie et pulsion de mort.

\section{La critique}

Le film de Gus Van Sant apporte un nouveau regard sur la question de la violence et de son esthétisation. Son traitement formel est très différent de ce que l'on est habitué à voir au cinéma : films à thèse ultradémonstratifs dans leurs construction et montage (à l'instar de Michael Moore), débauche de violence gratuite type Scarface (Brian de Palma, USA, 1983), regain du cinéma d'horreur et mise en abyme du genre dans Scream (Wes Craven, USA, 1996), où deux lycéens inventent une machination pour éliminer un à un leurs camarades, comme dans un jeu. Elephant illustre un refus de toute spectacularisation et justification de la violence.

Les crises de la modernité sont responsables du développement de ces différentes formes de violence dans lesquelles des dimensions identitaires et sociales se manifestent de façon aiguë. Les frustrations déclenchées par la relation déçue aux promesses des sociétés modernes (communication, progrès scientifiques et économiques, quête du bonheur,

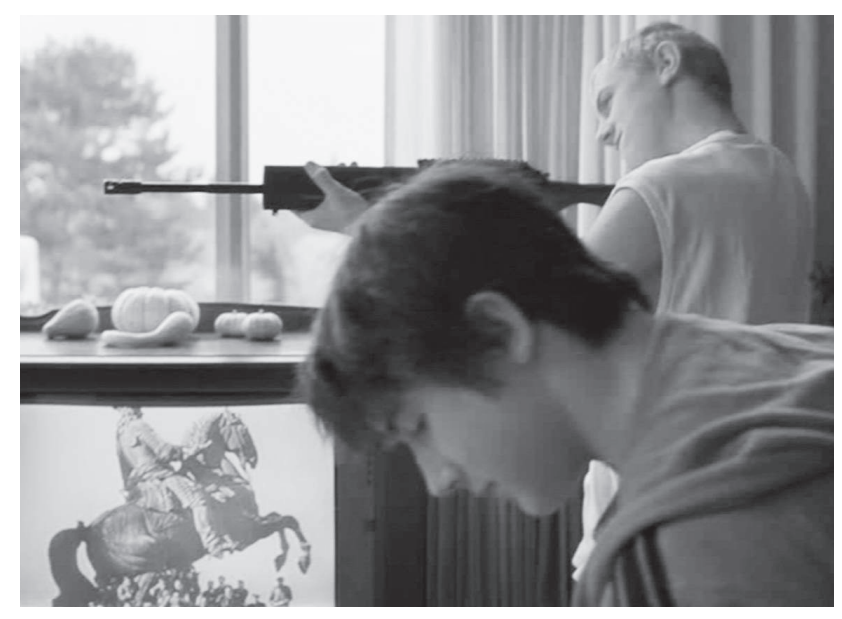


spectacle de la consommation ininterrompue,...) s'intensifient. Le lieu d'Elephant, c'est le lycée, c'est le sanctuaire de la modernité. Mais au lieu d'être un espace privilégié d'éducation, d'émancipation, de réciprocité, il concentre surtout des psychologies déstructurées et isolées les unes des autres, qui n'attendent que d'exploser. Car la crise a d'abord atteint la sphère pré-politique. L'homme moderne n'a plus d'autre solution que d'exprimer «son mécontentement envers le monde et son dégoût pour les choses telles qu'elles sont» en refusant d'en assumer la responsabilité pour les enfants:

"C’est comme si, chaque jour, les parents disaient: «En ce monde, même nous ne sommes pas en sécurité chez nous; comment s'y mouvoir, que savoir, quel bagage acquérir sont pour nous aussi des mystères. Vous devez essayer de faire de votre mieux pour vous en tirer; de toute façon vous n'avez pas de comptes à nous demander. Nous sommes innocents, nous nous lavons les mains de votre sort . $\gg \mathbf{1 3}$

Le projet moderne reposait sur la capacité pour l'individu d'apparaître libre face aux autres, détaché de sa soumission aux préjugés, aux croyances, en mesure d'exercer son jugement en public. Dans Elephant, l'humanité de l'homme semble avoir disparu au profit d'un monde sans signification, sans relation, sans responsabilité. C'est en construisant son film sur le maintien de l'oscillation entre modernité et romantisme que Gus Van Sant s'éloigne de la spectacularisation de la violence au profit d'une forme qui revendique la tension trouble entre deux visions du monde dont on ne saisit toujours pas suffisamment la portée et les significations. 